\title{
Prospective evaluation of simply modified MODS assay: an effective tool for TB diagnosis and detection of MDR-TB
}

Boonchai Chaiyasirinroje $e^{1, *}$ Myo Nyein Aung $2,3, *$

Saiyud Moolphate ${ }^{1,4}$ Yuthichai Kasetjaroen ${ }^{5}$ Somsak Rienthong ${ }^{5}$ Dhanida Rienthong ${ }^{5}$ Oranuch Nampaisan' Supalert Nedsuwan ${ }^{6}$ Wiravoot Sangchun ${ }^{6}$ Narin Suriyon ${ }^{7}$

Satoshi Mitarai ${ }^{4}$

Norio Yamada ${ }^{4}$

'TB/HIV Research Project, RIT, Chiang Rai, Thailand; ${ }^{2}$ Department of Public Health, Graduate School of Medicine, Juntendo University, Tokyo, Japan; ${ }^{3}$ Boromrajonani College of Nursing Nakhon Lampang (BCNLP), Lampang, Thailand; ${ }^{4}$ Research Institute of Tuberculosis (RIT), Tokyo, Japan; ${ }^{5}$ Bureau of Tuberculosis, Bangkok, Thailand; ${ }^{6}$ Chiang Rai Regional Hospital, Chiang Rai, Thailand; ${ }^{7}$ Chiang Rai Provincial Health Office, Chiang Rai, Thailand

*These authors contributed equally to this work
This article was published in the following Dove Press journal:

Infection and Drug Resistance

5 May 2012

Number of times this article has been viewed

Background and setting: Thailand is one of the highest tuberculosis (TB)-burdened countries. Chiang Rai, the northernmost province of Thailand has high tuberculosis and human immunodeficiency virus (HIV) prevalence and the laboratory workload for TB culture and drug susceptibility testing is increasing.

Objectives: To evaluate the simply modified microscopic-observation drug-susceptibility assay (MODS) in the setting of a developing country.

Methods: In this cross-sectional diagnostic study, a total of 202 sputum samples of clinically diagnosed TB patients were used to test the performance of MODS assay in reference to gold standard BACTEC ${ }^{\text {TM }}$ MGIT ${ }^{\text {TM }} 960$ liquid culture system and Ogawa solid culture. Sputum samples were collected from clinically diagnosed TB patients. Culture growth rate and time to culture positivity were compared among three methods. Performance of modified MODS assay was evaluated for detection of mycobacterium drug resistance in reference to MGIT antimicrobial susceptibility test (AST).

Result: Median time to culture positivity by MODS, solid, and liquid culture were 12, 30, and 6 days respectively. Compared to the drug susceptibility test (DST) result of reference liquid culture, the sensitivity and specificity of MODS for detection of multidrug-resistant tuberculosis (MDR-TB) was $85.7 \%$ and $97.5 \%$ respectively. MODS assay has a positive predicative value of $80 \%$ and negative predictive value of $96.5 \%$ for isoniazid resistance, $70 \%$ and $100 \%$ for rifampicin resistance, and $66.7 \%$ and $99.1 \%$ for MDR-TB.

Conclusion: MODS is a highly effective screening test for detection of MDR-TB.

Keywords: tuberculosis, drug resistance, MODS assay, Chiang Rai, Thailand

\section{Introduction}

Tuberculosis (TB) is a global disease with 9.5 million reported incident cases in 2009. 1,2 By 2009, multidrug-resistant tuberculosis (MDR-TB) prevalence had increased and nearly half a million cases have been reported worldwide. ${ }^{1}$ Thus, along with TB diagnosis, drug susceptibility testing (DST) has become an urgent clinical requirement for timely constitution of proper and effective TB treatment. However, TB diagnostic and laboratory capacity are still poor in many areas of highly TB-burdened countries. ${ }^{2}$ It causes a crucial barrier for detection of human immunodeficiency virus (HIV)associated and drug-resistant tuberculosis. ${ }^{3}$

Thailand is one of the 22 most TB-burdened countries, having an estimated 130,000 TB cases (182/100,000 population) in $2010 .^{2}$ It has the highest HIV prevalence in the Southeast Asian region, which was reported to affect $1.4 \%$ of the general population in 2009. ${ }^{3}$ It also has the highest MDR-TB prevalence in the Southeast
Correspondence: Norio Yamada Research Institute of Tuberculosis, Japan Anti-Tuberculosis Association, Japan Email nyamada@jata.or.jp 
Asian region, reported as 34.5\% (28.2\%-41.5\%) MDR among previously treated cases. ${ }^{4}$ The impact of TB-HIV dual epidemics has resulted in a high specimen workload for mycobacterial laboratories that exceeds human and technical resources.

The conventional solid culture method is slow and time consuming despite its high specificity. The highly accurate automated liquid culture method is rather costly for the developing world with a high TB burden. Therefore, a feasible, rapid, and sensitive TB culture and DST method applicable in a resource-challenged, high TB-burdened setting is an urgently needed priority.

Microscopic-observation drug-susceptibility assay (MODS), a noncommercial TB culture and DST method, is recommended by World Health Organization (WHO). ${ }^{5,6}$ It is cheaper and has shorter turn-around time than conventional gold standard methods. ${ }^{7}$ It has been validated in Peru and evaluated in some developing countries. ${ }^{6-13}$ Drug resistance can be detected within 1-2 weeks even with low establishment cost and technical needs. ${ }^{8}$ Therefore, it can be a reasonable solution for strengthening the TB diagnostic in developing countries.

In this study, we have made two simple modifications to MODS to satisfy completeness, safety, and less human resource needs, while conserving all the original culture technique. ${ }^{14}$ The first modification was the addition of paranitrobenzoic acid (PNB) for identification of TB and non-TB mycobacteria. The second was modification of the reading process by using a digital microscope kept in a biosafety cabinet. The objective of this prospective study was to compare the culture growth rate of modified MODS with conventional methods and validate its performance for detection of drug resistance in the practical setting of Chiang Rai, a TB-HIV-endemic area in northern Thailand on the border of Myanmar and Laos.

\section{Patients, materials, and methods Ethics}

The protocol of this study has been approved by Chiang Rai Regional Hospital Ethics Committee Thailand. The study was approved by the Bureau of Tuberculosis, Ministry of Public Health, Thailand.

\section{Study population and setting}

The study included clinical specimens according to inclusion criteria and single specimen per case basic. Clinical specimens mean sputum specimens of diagnosed cases at TB clinics of study site hospitals. A total of 202 clinically diagnosed TB patients were recruited prospectively at TB clinics in 17 hospitals in Chiang Rai province from January 2010 to June 2010. All the MODS assay, automated liquid culture by BACTEC ${ }^{\text {тм }}$ MGIT $^{\text {тM }}$ 960 (Becton, Dickinson and Company, Franklin Lakes, NJ) system and Ogawa solid culture were done at the mycobacterial laboratory of Chiang Rai Regional Hospital. A DST reference test by MGIT 960 was done at the National TB Reference Laboratory, a WHO Supranational Laboratory (SRL) in Bangkok serving four countries in Southeast Asia.

Sputum samples were collected by the time TB diagnosis was made clinically by physicians. Only the sputum samples meeting the following inclusion criteria were used for the study: (1) all pulmonary TB cases according to WHO criteria; (2) both acid-fast bacillus (AFB) smear positive and negative cases; and (3) both HIV-positive and -negative cases. Exclusion criteria were: (1) extrapulmonary TB; (2) age under 18 years; (3) sputum specimen collected more than 7 days after starting the anti-TB drugs; (4) sputum sample less than $2 \mathrm{~mL}$; and (5) sputum sample transported to mycobacterial laboratory without proper packaging, such as released screw cap of sputum container inside the plastic bag with zip-top.

\section{Storage of the sputum sample}

Sputum samples were properly stored at $2^{\circ} \mathrm{C}-8^{\circ} \mathrm{C}$ in a household refrigerator after collection. Samples were cultured within 7 days of collection. The median duration of storage was 4 days.

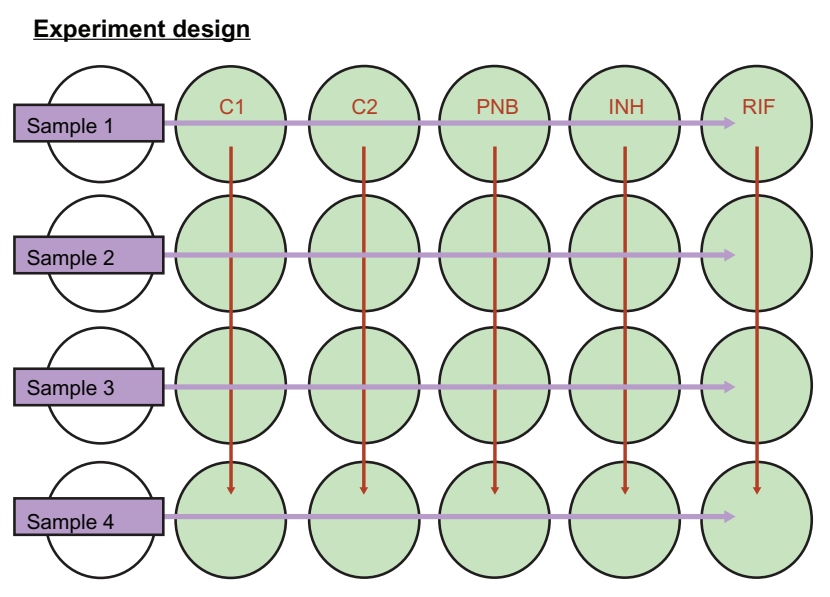

Figure I Design of MODS culture plates containing one well for PNB.

Abbreviations: $\mathrm{Cl}, \mathrm{C2}$ are culture wells $\mathrm{I}$ and 2; MODS, microscopic-observation drug-susceptibility assay; PNB, para-nitrobenzoic acid identification well; INH, isoniazid well for INH susceptibility test; RIF, rifampicin susceptibility test well. 

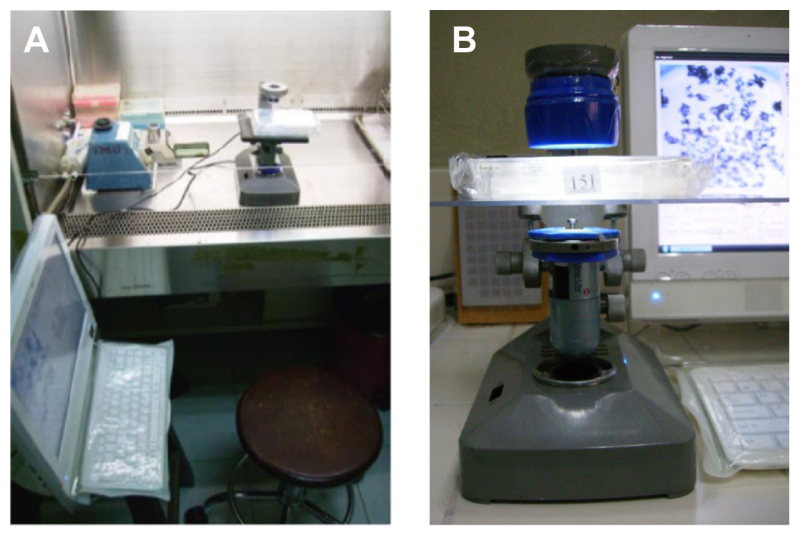

Figure 2 (A) Reading system of MODS plate inside bio-safety cabinet and (B) AM3I3T Dino-Lite PLUS Digital Microscope at viewer side of inverted microscope. Abbreviation: MODS, microscopic-observation drug-susceptibility assay.

\section{MODS}

All the MODS culture and drug susceptibility tests were carried out by the same technician who had more than 10 years of mycobacterial culture experience and a self-learned skill for MODS assay developed from MODS guidelines. ${ }^{14}$

An inverted microscope modified with a digital camera connected to a personal computer (PC) was used to examine 24-well MODs culture plates. Six culture wells were used for each sample: two wells for culture, one well with PNB, one well with isoniazid (INH), and another well with rifampicin. The Middlebrook 7 H9 Broth (Sigma-Aldrich, St Louis, MO) culture was used. All the steps of MODS assay developer's guideline were followed except the amount of decontaminated sputum used to inoculate in each well of a culture plate was reduced. ${ }^{14,15}$ We used $500 \mu \mathrm{L}$ instead of $750 \mu \mathrm{L}$. We have added two modifications as follows.

Modification 1: the addition of PNB test for identification of non-TB mycobacteria (NTM) PNB can differentiate Mycobacterium tuberculosis complex and NTM. ${ }^{16}$ To obtain the culture, identification, and DST results from a one MOD package at a time, we tested the addition of a PNB culture well in each line for the identification of M. tuberculosis and NTM. All five wells in one line were used for one sputum sample (Figure 1). One well in each line identified TB and NTB by using PNB. The PNB concentration in the well was $0.5 \mathrm{mg} / \mathrm{mL} .^{16}$

Modification 2: Use of computer-assisted digital camera for reading phase to improve biosafety and to reduce human resource need for frequent reading. The modified microscope and culture plates were kept in a biosafety-level 2 A cabinet (SCV-1305EC2AB [class II B]; Hitachi, Tokyo, Japan) as it is smaller than the original microscope for MODS. The reading was not necessarily to perform by direct observation through the eyepiece of laboratory technician. The inverted microscope was modified by connecting to a digital microscope

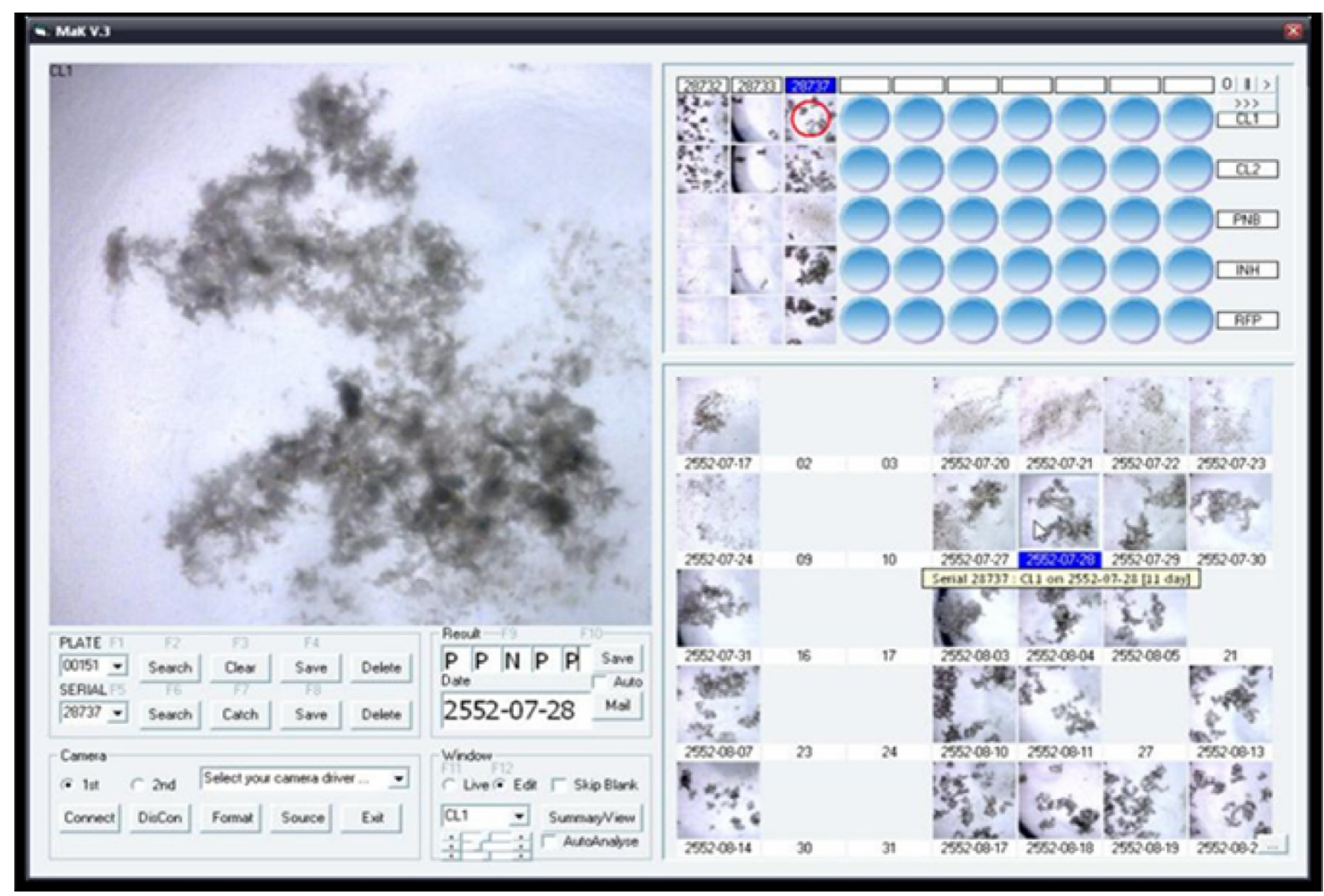

Figure 3 Daily reading and recording using digital microscope and software. 


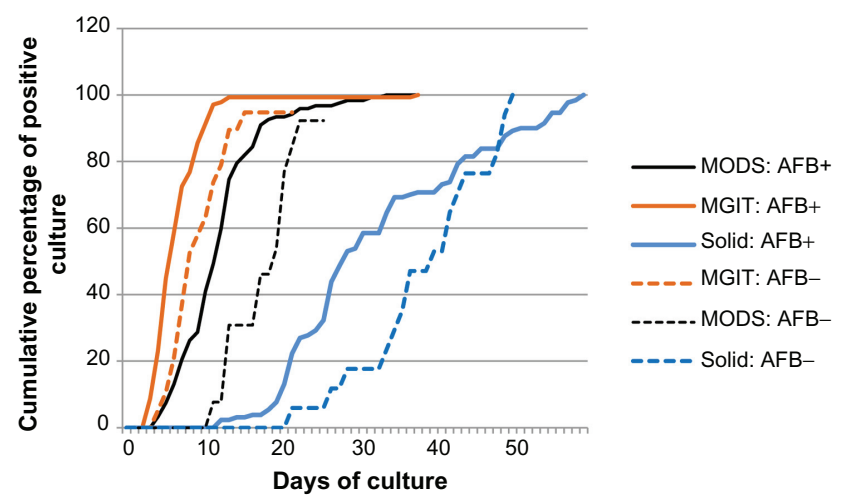

Figure 4 Cumulative culture positive rates of MODS assay, automated liquid culture by BACTEC TM MGIT TM 960 (Becton, Dickinson and Co, NJ, NY) and solid culture by Ogawa media.

Abbreviations: AFB+, acid fast bacilli positive sputum samples; AFB-, acid fast bacilli negative sputum samples; MODS, microscopic -observation drug susceptibility assay.

with 50× magnification on its viewer side. A Dino-Lite PLUS AM313T Digital Microscope (AnMo Electronics Corporation, New Taipei City, Taiwan) (Figure 2A and B) was used for this modification. The digital microscope was connected to a computer. We used $50 \times$ magnifications to capture an image of one whole MODS culture well at a time. One whole plate could be read with 24 shots.

\section{Reading and recording}

It was compulsory to read the control plate before reading each batch of MODS culture sample plates. All the samples of a culture batch were discarded if the control plate result was not valid. A positive result was decided by seeing growth in digital images on successive days (Figure 3). A positive result was decided by observation of the appearance of new growth compared with a clear culture well in the digital images taken previously. New appearance was defined as any pattern of growth. Negative culture was made sure by absence of growth after 35 days of reading.

Only after the growth had been seen in C1 and C2 wells, PNB-, isoniazid-, and rifampicin-containing wells were checked compulsorily (see Figure 1). Each culture plate was read by keeping an inverted light microscope inside the biosafety-level 2A cabinet. Results were read every day except during holidays and recorded in the logbook and computer software developed by the project. Positivity was judged by the same technician looking at pictures captured by the system and shown on the laptop computer monitor. Each specimen was determined by two technologists. The consensus between two opinions was checked by a statistician and unmatched results were repeated and concurred between two technologists. MODS culture results were judged independently, and MGIT results were blinded for individual specimens.

\section{Drug susceptibility tests}

For the purposes of quality control, a line of negative control wells was used in every plate. A positive control was prepared with a separate plate as a daily routine of MODS procedure. A negative control column of four wells on each plate was made to be sure that cross-contamination was not occurring (see Figure 1). The positive controls were run on a separate plate at the end of every day to check that (a) the media supported growth normally, and (b) the concentrations of rifampicin $(1 \mu \mathrm{g} / \mathrm{mL})$ and isoniazid $(0.1 \mu \mathrm{g} / \mathrm{mL})$ were correct. ${ }^{17} \mathrm{INH}$ concentration $0.1 \mu \mathrm{g} / \mathrm{mL}$ and rifampicin $1 \mu \mathrm{g} / \mathrm{mL}$ solution were used in the drug susceptibility test.

\section{Solid and liquid culture}

MGIT 960 automated liquid culture method

This is the gold standard and reference method in this study. The manufacturer's instruction was strictly followed for the BACTEC ${ }^{\mathrm{TM} M G I T} 960^{\mathrm{TM}}$ automated TB liquid culture system. The culture inoculation was done at the mycobacterial laboratory of Chiang Rai Regional Hospital. The MGIT AST

Table I Characteristic of the pulmonary TB patients at the study entry

\begin{tabular}{lll}
\hline Characteristics & Number & Percentage \\
\hline $\begin{array}{l}\text { Number } \\
\text { Sex }\end{array}$ & 202 & 100 \\
Male & 155 & 76.73 \\
Female & 47 & 23.27 \\
Age (years) & & \\
Median (IQR) & $48(37-59)$ & \\
Race & & \\
Thai & 117 & 57.92 \\
Hill tribe & 50 & 24.75 \\
Myanmar and Lao & 35 & 17.33 \\
Type of patient & & \\
IPD & 108 & 53.47 \\
OPD & 87 & 43.07 \\
Unknown & 7 & 3.47 \\
Type of TB treatment & & \\
New case & 178 & 88.12 \\
Re-treatment & 24 & 11.88 \\
Sputum AFB status at TB diagnosis & \\
Pulmonary AFB+ & 147 & 72.77 \\
Pulmonary AFB scanty & 12 & 5.94 \\
Pulmonary AFB- & 43 & 21.29 \\
\hline
\end{tabular}

Abbreviations: AFB, sputum acid-fast bacillus smears; IPD, admitted as inpatient at the hospital; IQR, interquartile range; OPD, treated at TB clinic, outpatient department; TB, tuberculosis. 
Table 2 Median time to culture growth in three different TB culture methods: Ogawa solid culture, BACTECTM MGITTM 960 liquid culture system and MODS assay in sputum samples of clinically diagnosed TB patients, Chiang Rai, Thailand 2010

\begin{tabular}{|c|c|c|c|c|c|c|}
\hline \multirow[t]{2}{*}{$N=202$ cases } & \multicolumn{2}{|c|}{ Culture growth } & \multirow{2}{*}{$\begin{array}{l}\text { Median time to culture } \\
\text { positivity (days) }\end{array}$} & \multirow{2}{*}{$\begin{array}{l}\text { Interquartile } \\
\text { range }\end{array}$} & \multirow{2}{*}{$\begin{array}{l}\text { Minimum/ } \\
\text { maximum }\end{array}$} & \multirow[t]{2}{*}{$P$ value } \\
\hline & $(n / N)$ & $(\%)$ & & & & \\
\hline \multicolumn{7}{|l|}{ Solid culture } \\
\hline AFB-positive & $130 / 147$ & 88.44 & 28 & $22-42$ & $12-58$ & $<0.001^{\mathrm{s}}$ \\
\hline AFB-negative & $17 / 55$ & 30.91 & 39 & $34-43$ & $21-49$ & $<0.001^{\mathrm{s}}$ \\
\hline Total & I47/202 & 72.77 & 30 & $23-42$ & $12-58$ & $<0.001^{\mathrm{s}}$ \\
\hline \multicolumn{7}{|l|}{ Liquid culture } \\
\hline AFB-positive & $138 / 147$ & 93.88 & 6 & $5-8$ & $3-37$ & $<0.00 I^{\mathrm{L}}$ \\
\hline AFB-negative & $19 / 55$ & 34.55 & 8 & $7-12$ & $4-22$ & $<0.00 I^{\mathrm{L}}$ \\
\hline Total & $157 / 202$ & 77.72 & 6 & $5-9$ & $3-37$ & $<0.00 I^{\mathrm{L}}$ \\
\hline \multicolumn{7}{|l|}{ MODS } \\
\hline AFB-positive & $122 / 147$ & 82.99 & 12 & $8-14$ & $4-33$ & \\
\hline AFB-negative & $13 / 55$ & 27.67 & 19 & $13-20$ & $11-26$ & \\
\hline Total & $135 / 202$ & 66.83 & 12 & $9-15$ & $4-33$ & \\
\hline
\end{tabular}

Notes: $\mathrm{n}=$ number of positive growth; $\mathrm{N}=$ total number; ${ }^{\mathrm{S} P}$ value for comparison of microscopic-observation drug-susceptibility assay (MODS) versus solid; ${ }^{\mathrm{P}} \mathrm{P}$ value for comparison of MODS versus liquid. Growth means culture positive by seeing growth.

tests were performed at National TB Reference Laboratory of Thailand in Bangkok.

Ogawa solid culture

This is the routine culture method currently used in Chiang Rai. Solid cultures were inoculated in parallel with MODS and MGIT at the mycobacterial laboratory, Chiang Rai Regional Hospital. The routine protocol of the Chiang Rai Laboratory was followed for solid culture in Ogawa media.

\section{Statistical analysis}

Stata software (version 11; StataCorp LP, College Station, TX) was used for statistical data analysis. The Mann-Whitney $U$ test was used for comparing median time to positive culture growth between MODS versus each two methods. DST performance of MODS assay was validated in reference to the gold standard MGIT 960 and parameters were computed by Stata software.

\section{Results}

Altogether there were 202 sputum samples in this study, which comprised $72.77 \%$ of the smear AFB-positive sputum samples. Table 1 shows the characteristics of TB patients in the study population. Most of the cases were new cases $(88.12 \%)$. Moreover, $16.34 \%$ of the studied participants were HIV-coinfected.

\section{Time to culture positivity}

Median time to culture positivity was 12 days by MODS, 6 days by MGIT 960 automated liquid culture, and 30 days by Ogawa solid culture. MODS assay has a shorter median time to culture positivity than solid culture, but longer than the automated liquid culture (Table 2). The contamination rate of MODS is higher than that of liquid and solid culture. The contamination rates of the MODS, MGIT, and Ogawa methods were $15.8 \%$ (32 samples), 11.9\% (24 samples), and $10.9 \%$ (22 samples), respectively. Contamination rate in smear positive samples were 3.4\% in MGIT, 6.12\% in solid culture and $10.2 \%$ in MODS whereas contamination rate in smear negative sample were $23.64 \%$ in solid culture, $30.91 \%$ in MODS and 34.55\% in MGIT. Moreover, culture growth rates were $71.14 \%$ in $\mathrm{HIV}$-positive patients and $82.35 \%$ in HIV-negative patients.

Table 3 DST performance result of MODS in reference to BACTEC TM MGITTM 960 automated liquid culture for detection of drugresistant $M$. tuberculosis in sputum

\begin{tabular}{|c|c|c|c|c|c|c|}
\hline \multirow[t]{3}{*}{ MODS } & \multicolumn{6}{|c|}{ DST by MGIT } \\
\hline & \multicolumn{2}{|l|}{ Rifampicin } & \multicolumn{2}{|l|}{ Isoniazid } & \multicolumn{2}{|l|}{ MDR-TB } \\
\hline & Resistance & Sensitivity & Resistance & Sensitivity & Resistance & Sensitivity \\
\hline Resistance & 7 & 3 & 12 & 3 & 6 & 3 \\
\hline Sensitivity & 0 & 119 & 4 & II I & I & 116 \\
\hline Total & 7 & 122 & 16 & $1 \mid 4$ & 7 & 119 \\
\hline
\end{tabular}

Abbreviations: DST, drug susceptibility test; MODS, microscopic-observation drug-susceptibility assay; MDR-TB, multidrug-resistant tuberculosis. 
Table 4 Performance characteristic MODS assay compared to reference BACTECTM MGITTM 960 automated liquid culture for detection of drug-resistant M. tuberculosis in sputum, Chiang Rai, Thailand, 2010

\begin{tabular}{llll}
\hline & Rifampicin & Isoniazid & MDR-TB \\
\hline Number of cases & 129 & 130 & 126 \\
Kappa value & 0.8115 & 0.7437 & 0.7333 \\
Agreement & $97.67 \%$ & $94.62 \%$ & $96.83 \%$ \\
Area under ROC & $0.988(0.974-1)$ & $0.862(0.751-0.972)$ & $0.916(0.775-1)$ \\
Sensitivity (95\% Cl) & $100(59-100)$ & $75(47.6-92.7)$ & $85.7(42.1-99.6)$ \\
Specificity (95\% Cl) & $97.5(93-99.5)$ & $97.4(92.5-99.5)$ & $97.5(92.8-99.5)$ \\
PPV (95\% Cl) & $70(34.8-93.3)$ & $80(51.9-95.7)$ & $66.7(29.9-92.5)$ \\
NPV (95\% Cl) & $100(96.9-100)$ & $96.5(91.3-99)$ & $99.1(95.3-100)$ \\
Likelihood ratio (+) & $40.7(13.3-12.4)$ & $28.5(9.01-90.2)$ & $34(10.7-108)$ \\
Likelihood ratio (-) & 0 & $0.257(0.11-0.6)$ & $0.147(0.024-0.9)$ \\
\hline
\end{tabular}

Abbreviations: $\mathrm{Cl}$, confidence interval; MODS, microscopic-observation drug-susceptibility assay; MDR-TB, multidrug-resistant tuberculosis; NPV, negative predictive value; PPV, positive predictive value; ROC, receiver-operating characteristic curve.

\section{Performance of MODS for detection of drug resistance in clinical sample}

The performance of the MODS assay in clinical sample was compared with the reference MGIT 960 result for detection of mycobacterial drug resistance (Table 3). Of the 129 cases tested for rifampicin resistance, the MODS assay was in agreement with the MGIT 960 system in 126 cases. The positive predicative value (PPV) was 70\% (95\% confidence interval [CI]: 34.8-93.3) and the negative predictive value (NPV) was $100 \%$ (95\% CI: 96.9-100). Of the 130 cases tested for INH resistance, MODS assay was in agreement with the MGIT 960 AST result in only 123 cases. The PPV of MODS for INH resistance was $80 \%$ (95\% CI: 51.9-95.7) and the NPV was $96.5 \%$ (95\% CI: 91.3-99\%). Of the 126 specimens tested for MDR, MODS assay was in agreement with the MGIT 960 AST result in 122 cases (Table 3). The PPV for detection of MDR-TB was $66.7 \%$ (95\% CI: 29.9-92.5) and NPV was 99.1\% (95\% CI: 95.3-100) (Table 4).

Negative predicative values in detection of INH resistance, rifampicin resistance, and MDR-TB were consistently high. Positive likelihood ratio for detection of rifampicin resistance (40.7), INH resistance (28.5), and MDR-TB (34) indicated that MODS assay would be an effective DST for ruling out the mycobacterial drug resistance (Table 4).

\section{Identification of mycobacterium species by PNB}

In this study, MODS assay could identify only one case of NTM infection. Reference method PNB on L-J media identified six NTM cases out of 202. MODS results agreed with reference method in $98.28 \%$ (114/116) of samples tested for NTM idetification, but there was no positive result agreed between two methods.

\section{Discussion}

A TB culture method which has fast turnaround time and a rapid DST result with a reasonable price is an operational need in the TB control program in Chiang Rai and many areas with similar tuberculosis burdens across the world. MODS assay is a reasonable candidate by existing evidences.

Two meta-analyses have reported that MODS can outperform other non-commercial methods of DST. ${ }^{5,18}$ However, Banerjee et a ${ }^{19}$ point out the weakness of meta-analyses as lack of studies in developing countries. In the current study, we have tested the performance of MODS in a study population entirely composed of clinically diagnosed TB patients' sputum samples. This study was a prospective study conducted in a real clinical setting in a highly TB-burdened area with limited resources. All processes involved with sputum sample collection, sputum specimen transport, storage, and processing were conducted through a routine TB control program service at 16 district hospitals and a regional hospital. Therefore, our study finding is expected to provide existing and growing evidence for TB diagnostic pipe lines following research from these routine processes.

The culture growth rate and time to culture positivity were compared to reference liquid culture and Ogawa solid culture. We have seen faster median time to culture growth of MODS assay than in Ogawa solid culture. However, MODS assay is longer than automated liquid culture. A community based study in Peru has demonstrated that the growth rate of MODS is faster than that of the MGIT liquid culture at 7 days and 13 days, respectively. ${ }^{7}$ Clinical studies from Ethiopia reported that the median turn-around time of smear AFB-positive sputum samples by MODS culture was 9 days and the growth rate was $96.9 \%(n=262){ }^{8}$ Our finding about the culture growth 
rate and time to culture positivity of MODS is rather different from those previous findings. In the current study of simply modified MODS assay, the culture-growth rate of sputum smear AFB-positive samples was $82.99 \%$ and median time to detect culture growth was 12 days (see Table 2).

These culture performance results may be due to the use of a relatively smaller amount of decontaminated sputum $(500 \mu \mathrm{L})$ for inoculating in Middlebrook $7 \mathrm{H} 9$ Broth or, due to the modification of using digital microscope and computerized recording. Because the lens resolution is not very high, the colony growth may not be obviously seen in the early days of bacterial growth. The modification has improved safety, whereas in the earlier days of MODS there were biohazard concerns. ${ }^{20}$ Moreover, the tedious daily workload of checking the culture plates was also a major weak point. ${ }^{20}$

We have modified MODS to solve these issues. The reading process of MODS was modified by using a computer assisted digital camera. The potential advantages are: (1) all the culture material and microscope can be kept in a bio-safety cabinet, minimizing the chance of biohazard to laboratory workers, (2) the morphological changes in MODS culture wells can be checked more frequently with less human effort, (3) the morphology of culture wells can be compared day by day, (4) the culture results can be stored in digital copy, enabling sharing so that technical experts can review the results. In this study we used daily recording of the morphological changes in culture wells.

During the current study, similar kinds of reading system were reported by Zimic et al. ${ }^{21}$ However, their adjustable magnification was different from ours. Zimic et al used an artesenal inverted microscope with 60-100× amplifier for direct visualization of MODS cultures which aimed to capture a cording image. We used 50× magnifications to capture an image of one whole well at a time. It made the reading process faster. Identification by using PNB well was tested but did not work.

The diagnostic performance parameters of the MODS indicated that it could be a highly valuable screening tool for identifying MDR-TB. Negative predictive values of the test were consistently high for INH resistance, rifampicin resistance, and MDR-TB. In our study, a higher concentration of INH $0.1 \mu \mathrm{g} / \mathrm{mL}$ was used. Positive and negative control wells were carefully checked for quality of DST. However, area under the relative operating characteristic (ROC) curve for INH resistance was rather small at 0.862 compared to other previous studies..$^{5}$ Likewise, the meta-analysis by Bwanga et $\mathrm{al}^{5}$ has reported the good performance of MODS despite variable pooled sensitivity and specificity in detection of INH resistance. In the current study we found that modified MODS had lower sensitivity in detection of $\mathrm{INH}$ resistance than the pooled sensitivity reported by that meta-analysis. ${ }^{5}$ However, sensitivity is high for detection of rifampicin resistance which is a good proxy indicator of MDR-TB. Therefore, it would be a better option to use the MODS assay for screening drugresistant cases and confirm the positive results by using a more specific test. MODS is a nonpatented, low cost and low technology culture method. With our modification of reading process it would be a low workload culture method and therefore usable in resource-challenged settings.

\section{Limitation of the study}

We could not compare the time to result, total time taken from taking sample to giving culture and DST result back to clinician, in each method. The reference methods of DST were carried out at the National Reference Laboratory in Bangkok. Median time to result is 70 days for Chiang Rai province. In this study MODS assay has culture turnaround time of 12 days but we did not deliver the result to clinicians. So, we did not compare the time to result between MODS and reference method.

In our sample, $42 \%$ of the study population were non-Thai patients. The distance of hill tribe villages from the TB laboratory, the language barrier, and health illiteracy among such patients were also limitations in practice to get the sputum samples which met the eligibility criteria of the study.

We have calculated the sample size necessary for power of $80 \%$ with $95 \%$ CI based on prevalence of INH resistance in Chiang Rai and found it was 250 samples. After application of eligibility criteria, only 202 samples remained for analysis. To keep the internal validity of the study, we sacrificed some samples.

\section{Conclusion}

In a highly TB-burdened area like Chiang Rai, Thailand, a rapid, sensitive, and affordable culture method is operationally necessary. After applying a modification for better safety and averting human resource need by use of computer-assisted reading, MODS would be a safer and effective culture and DST method for screening multidrug-resistant tuberculosis.

\section{Acknowledgments}

This work was sponsored partly by Research Institute of Tuberculosis, Japan. David AJ Moore is acknowledged for his technical advice. Hideki Yani is acknowledged for his suggestions and research protocol comments. Nurses, 
medical technologists and TB clinic staff of 17 hospitals in Chiang Rai province are acknowledged for their cooperative assistance.

\section{Authors' contribution}

Norio Yamada, Myo Nyein Aung, Saiyud Moolphate and Boonchai Chaiyasirinroje designed the study. Myo Nyein Aung developed the research proposal which was edited by Norio Yamada, Satoshi Mitarai, Somsak Rienthong, and Dhanida Rienthong in October 2009. Myo Nyein Aung and Boonchai Chaiyasirinroje developed the standard operating procedures for the study in November 2009. Boonchai Chiyasirinrote carried out all the TB cultures and MODS procedures with the help of Oranuch Nampaisan. Supalert Nedsuwan, Wiravoot Sangchun, and Narin Suriyon contributed to the research process and collection of data. Saiyud Moolphate and Myo Nyein Aung analyzed the data and interpreted the results. Myo Nyein Aung wrote the manuscript. Norio Yamada, Somsak Rienthong, Yuthichai Kasetjaroen, Dhanida Rienthong, Satoshi Mitarai and Boonchai Chaiyasirinroje critically reviewed, revised, and amended the manuscript. All the authors contributed their comments which have been amalgamated into the final manuscript by Myo Nyein Aung.

\section{Disclosure}

The authors declare no conflict of interest in this work.

\section{References}

1. World Health Organization (WHO). Global tuberculosis control; 2010. Available from http://www.who.int/tb/publications/global_report/2010/ en/index.html. Accessed August 8, 2011.

2. World Health Organization (WHO). Global tuberculosis control; 2011. Available from: http://www.who.int/tb/publications/global_report/en/. Accessed October 24, 2011.

3. World Health Organization (WHO).TB diagnostics and laboratory strengthening; 2011. Available from: http://www.who.int/tb/laboratory/en/. Accessed August 8, 2011.

4. World Health Organization (WHO). Regional Report 2011: Tuberculosis control in Southeast Asain Region; 2011. Available from: http://www. searo.who.int/LinkFiles/TB_Day_Kit_TB_Annual_Report_2011.pdf. Accessed June 8, 2011.

5. Bwanga F, Hoffner S, Haile M, Joloba M. Direct susceptibility testing for multi drug resistant tuberculosis: a meta-analysis. BMC Infect Dis. 2009;9:67.
6. Moore DA, Mendoza D, Gilman RH, et al. Microscopic observation drug susceptibility assay, a rapid, reliable diagnostic test for multidrugresistant tuberculosis suitable for use in resource-poor settings. $J$ Clin Microbiol. 2004;42(10):4432-4437.

7. Moore DA, Evans CA, Gilman RH, et al. Microscopic-observation drug-susceptibility assay for the diagnosis of TB. $N$ Engl J Med. 2006;355(15):1539-1550

8. Shiferaw G, WoldeamanuelY, Gebeyehu M, Girmachew F, Demessie D, Lemma E. Evaluation of microscopic observation drug susceptibility assay for detection of multidrug-resistant Mycobacterium tuberculosis. J Clin Microbiol. 2007;45(4):1093-1097.

9. Michael JS, Daley P, Armstrong L, et al. Prospective evaluation of microscopic observation drug-susceptibility (MODS) assay for the diagnosis of active tuberculosis (TB) in India - preliminary analysis. Int J Infect Dis. 2008;12(Suppl 1):e324.

10. Mello FCQ, Arias MS, Rosales S, et al. Clinical evaluation of the microscopic observation drug susceptibility assay for detection of Mycobacterium tuberculosis resistance to isoniazid or rifampin. J Clin Microbiol. 2007;45(10):3387-3389.

11. Ejigu GS, Woldeamanuel Y, Shah NS, Gebyehu M, Selassie A, Lemma E. Microscopic-observation drug susceptibility assay provides rapid and reliable identification of MDR-TB. Int J Tuberc Lung Dis. 2008;12(3):332-337.

12. Arias M, Mello FC, Pavón A, et al. Clinical evaluation of the microscopic observation drug-susceptibility assay for detection of tuberculosis. Clin Infect Dis. 2007;44(5):674-680.

13. Limaye K, Kanade S, Nataraj G, Mehta P. Utility of microscopic observation of drug susceptibility (MODS) assay for Mycobacterium tuberculosis in resource constrained settings. Indian J Tuberc. 2010;57(4):207-212.

14. Coronel J, Roper M, Caviedes L, Moore D. MODS. A user guide. Microscopic-observation drug-susceptibility assay; 2008. Available from: http://www.modsperu.org/MODS_user_guide.pdf. Accessed October 12, 2009.

15. Moore DAJ. MODS laboratory accreditation; 2008. http://www. modsperu.org/MODS_laboratory_validation.pdf. Accessed October 12, 2009.

16. Giampaglia CMS, Martins MC, Chimara E, et al. Differentiation of Mycobacterium tuberculosis from other mycobacteria with rho-nitrobenzoic acid using MGIT960. Int J Tuberc Lung Dis. 2007;11(7):803-807.

17. Moore DAJ. MODS QC question from TB HIV research consortium Thailand. In: Aung MN, editor. 2009.

18. Minion J, Leung E, Menzies D, Pai M. Microscopic-observation drug susceptibility and thin layer agar assays for the detection of drug resistant tuberculosis: a systematic review and meta-analysis. Lancet Infect Dis. 2010;10(10):688-698.

19. Banerjee Y, Taranikanti V, Bayoumi R. Assays for drug resistant tuberculosis in high burden countries. Lancet Infect Dis. 2011;11(3): 161-162.

20. Palomino JC, Martin A, Portaels F. MODS Assay for the Diagnosis of TB. N Engl J Med. 2007;356(2):188-189.

21. Zimic M, Velazco A, Comina G, et al. Development of low-cost inverted microscope to detect early growth of Mycobacterium tuberculosis in MODS culture. PLoS ONE. 2010;5(3):e9577.
Infection and Drug Resistance

\section{Publish your work in this journal}

Infection and Drug Resistance is an international, peer-reviewed openaccess journal that focuses on the optimal treatment of infection (bacterial, fungal and viral) and the development and institution of preventive strategies to minimize the development and spread of resistance. The journal is specifically concerned with the epidemiology of antibiotic

\section{Dovepress}

resistance and the mechanisms of resistance development and diffusion in both hospitals and the community. The manuscript management system is completely online and includes a very quick and fair peerreview system, which is all easy to use. Visit http://www.dovepress.com/ testimonials.php to read real quotes from published authors. 